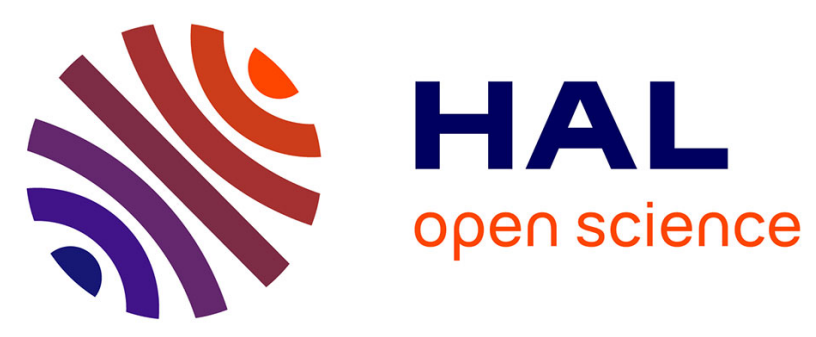

\title{
ECG Denoising using Angular Velocity as a State and an Observation in an Extended Kalman Filter Framework
}

\author{
Mahsa Akhbari, Mohammad B. Shamsollahi, Christian Jutten, Bertrand \\ Coppa
}

\section{- To cite this version:}

Mahsa Akhbari, Mohammad B. Shamsollahi, Christian Jutten, Bertrand Coppa. ECG Denoising using Angular Velocity as a State and an Observation in an Extended Kalman Filter Framework. EMBC 2012 - 34th Annual International Conference of the IEEE Engineering in Medicine and Biology Society, Aug 2012, San Diego, Californie, United States. pp.2897-2900. hal-00853756

\section{HAL Id: hal-00853756 https://hal.science/hal-00853756}

Submitted on 27 Sep 2013

HAL is a multi-disciplinary open access archive for the deposit and dissemination of scientific research documents, whether they are published or not. The documents may come from teaching and research institutions in France or abroad, or from public or private research centers.
L'archive ouverte pluridisciplinaire HAL, est destinée au dépôt et à la diffusion de documents scientifiques de niveau recherche, publiés ou non, émanant des établissements d'enseignement et de recherche français ou étrangers, des laboratoires publics ou privés. 


\title{
ECG Denoising using Angular Velocity as a State and an Observation in an Extended Kalman Filter Framework
}

\author{
Mahsa Akhbari ${ }^{1}$, Mohammad B. Shamsollahi ${ }^{1}$, Christian Jutten ${ }^{2}$ and Bertrand Coppa ${ }^{3}$
}

\begin{abstract}
In this paper an efficient filtering procedure based on Extended Kalman Filter (EKF) has been proposed. The method is based on a modified nonlinear dynamic model, previously introduced for the generation of synthetic ECG signals. The proposed method considers the angular velocity of ECG signal, as one of the states of an EKF. We have considered two cases for observation equations, in one case we have assumed a corresponding observation to angular velocity state and in the other case, we have not assumed any observations for it. Quantitative evaluation of the proposed algorithm on the MIT-BIH Normal Sinus Rhythm Database (NSRDB) shows that an average SNR improvement of $8 \mathrm{~dB}$ is achieved for an input signal of $-4 \mathrm{~dB}$.
\end{abstract}

Index Terms-Extended Kalman Filter (EKF), Angular velocity, Electrocardiogram (ECG), ECG Dynamical Model, Denoising

\section{INTRODUCTION}

The Electrocardiogram (ECG) is a non-invasive, safe and quick method for diagnosing cardiovascular diseases. ECG signals are usually corrupted with unwanted interferences. The extraction of pure cardiological indices from noisy measurements, has been one of the major concerns of biomedical signal processing. Despite the rich literature in this field, there are still many unsolved problems, which need to benefit from signal processing developments.

Recently, Bayesian filters were proposed for ECG denoising [1] and filtering cardiac contaminants [2]. The state space model used in these approaches was inspired from the model proposed by McSharry et al., who suggested the use of Gaussian Mixture Model (GMM) to generate synthetic ECGs [3]. It was later found that by some modifications, the filtering framework developed by Clifford et al. [4] and Sameni et al. [1], could be used as a parameter-based framework for model-based ECG filtering, simultaneous denoising, compression and beat segmentation [5], [6].

In previous works, a global angular velocity $(\omega)$ using the average RR-interval of the whole signal was used which is not precise, especially for long ECG signals or signals with major RR-interval deviations. It should be noted however, that $\omega$ can also be considered to vary on an intra-beat basis due to heart rate and RR-interval variations. In this work, we would like to investigate if using the angular velocity

1 M. Akhbari and M. B. Shamsollahi are with Department of Electrical Engineering, Sharif university of Technology, Tehran, Iran mahsa_akhbari@ee.sharif.edu, mbshams@sharif.edu

${ }^{2} \mathrm{C}$. Jutten is with the GIPSA-Lab, Grenoble and with Institut Universitaire de France, France firstname.name@gipsa-lab.grenoble-inp.fr

${ }^{3} \mathrm{~B}$. Coppa is with CEA-LETI, Minatec Campus, Grenoble, France. variable is able to improve ECG estimation, especially in noisy cases.

Sameni et al. [1] have proposed an Extended Kalman Filter (EKF) algorithm for denoising ECG signals (called EKF2). They have considered two state variables and two corresponding observations. After that Sayadi et. al [5] extended EKF2 framework and added parameters of ECG dynamical model as states to EKF2 and introduced "EKF17" algorithm which was used for ECG denoising and compression. They also described a Gaussian wave-based state space model [6] in which each characteristic waves, i.e. P, QRS and T, have been considered as states. Most recently, Lin et. al [7] considered 18 state variables (state variables of EKF17 and angular velocity as a new state variable) and two observations (noisy ECG signal and its roughly estimated phase) in their model and they used marginalized particle filter for tracking ECG using this modified nonlinear state-space model. In this paper we consider angular velocity of ECG as third state variable and its roughly approximation as third observation in an Extended Kalman Filter framework.

Basics of EKF is discussed in Section II. In Section III, we explain our proposed method for denoising ECG signals. "EKF2","EKF3" and "EKF3-2" algorithms are described in this section. In section IV, we present the results of applying the proposed method to a real ECG dataset. Finally, discussion and conclusions are provided in Section V.

\section{BAsics of Extended Kalman Filter}

The Extended Kalman Filter (EKF) is a nonlinear extension of conventional Kalman Filter (KF) that has been specifically developed for systems having nonlinear dynamic models [8]. For a discrete nonlinear system with the state vector $\underline{x}_{k}$ and observation vector $\underline{y}_{k}$, the dynamic model may be formulated as follows:

$$
\left\{\begin{array}{l}
\underline{x}_{k}=f\left(\underline{x}_{k-1}, \underline{w}_{k}, k\right) \\
\underline{y}_{k}=g\left(\underline{x}_{k}, \underline{v}_{k}, k\right)
\end{array}\right.
$$

where $\underline{w}_{k}$ and $\underline{v}_{k}$ are the process and measurement noises respectively with covariance matrices $Q_{k}=E\left\{\left(\underline{w}_{k}-\underline{\bar{w}}_{k}\right)\left(\underline{w}_{k}-\right.\right.$ $\left.\left.\underline{\bar{w}}_{k}\right)^{T}\right\}$ and $R_{k}=E\left\{\left(\underline{v}_{k}-\bar{v}_{k}\right)\left(\underline{v}_{k}-\bar{v}_{k}\right)^{T}\right\}$ which $\underline{\bar{w}}_{k}=E\left\{\underline{w}_{k}\right\}$ and $\underline{\bar{v}}_{k}=E\left\{\underline{v}_{k}\right\}$. The initial state estimate of the state $\underline{x}_{0}$ is defined with $\underline{\bar{x}}_{0}=E\left\{\underline{x}_{0}\right\}$ and $P_{0}=E\left\{\left(\underline{x}_{0}-\underline{\bar{x}}_{0}\right)\left(\underline{x}_{0}-\underline{\bar{x}}_{0}\right)^{T}\right\}$. In order to use a Kalman Filter for this system, it is necessary to derive a linear approximation of (1) near desired reference points $\left(\underline{\hat{x}}_{k-1}, \underline{\bar{w}}_{k}\right)$ and $\left(\underline{\hat{x}}_{k}^{-}, \underline{\bar{v}}_{k}\right)$. This approximation will lead 
to the following linear estimate:

$$
\left\{\begin{array}{l}
\underline{x}_{k} \approx f\left(\underline{\hat{x}}_{k-1}, \overline{\bar{w}}_{k}, k\right)+A_{k}\left(\underline{x}_{k-1}-\underline{\hat{x}}_{k-1}\right)+F_{k}\left(\underline{w}_{k}-\bar{w}_{k}\right) \\
\underline{y}_{k} \approx g\left(\underline{\hat{x}}_{k}^{-}, \underline{\bar{v}}_{k}, k\right)+C_{k}\left(\underline{x}_{k}-\underline{\hat{x}}_{k}^{-}\right)+G_{k}\left(\underline{v}_{k}-\underline{\bar{v}}_{k}\right)
\end{array}\right.
$$

where

$$
\begin{aligned}
& A_{k}=\left.\frac{\partial f\left(\underline{x}, \underline{\bar{w}}_{k}, k\right)}{\partial \underline{x}}\right|_{\underline{x}=\underline{\hat{x}}_{k-1}}, F_{k}=\left.\frac{\partial f\left(\underline{\hat{x}}_{k-1}, \underline{w}, k\right)}{\partial \underline{w}}\right|_{\underline{w}=\underline{\bar{w}}_{k}} \\
& C_{k}=\left.\frac{\partial g(\underline{x}, \underline{\underline{\bar{s}}}, k)}{\partial \underline{x}}\right|_{\underline{x}=\underline{\hat{x}}_{k}^{-}}, G_{k}=\left.\frac{\partial g\left(\underline{\hat{x}}_{k}^{-}, \underline{v}, k\right)}{\partial \underline{v}}\right|_{\underline{v}=\underline{\bar{v}}_{k}}
\end{aligned}
$$

In order to implement the EKF, the time propagation is done using the original nonlinear equation, while the Kalman Filter gain and the covariance matrix are calculated from the linearized equations, summarized as follows:

$$
\begin{aligned}
& \hat{\hat{x}}_{k}^{-}=f\left(\underline{\hat{x}}_{k-1}, \bar{w}_{k}, k\right) \\
& P_{k}^{-}=A_{k} P_{k-1} A_{k}^{T}+F_{k} Q_{k} F_{k}^{T}
\end{aligned}
$$

and the measurement propagation equations are:

$$
\begin{aligned}
& \underline{\hat{x}}_{k}=\underline{\hat{x}}_{k}^{-}+K_{k}\left[\underline{y}_{k}-g\left(\underline{\hat{x}}_{k}^{-}, \underline{\underline{v}}_{k}, k\right)\right] \\
& K_{k}=P_{k}^{-} C_{k}^{T}\left[C_{k} P_{k}^{-} C_{k}^{T}+G_{k}\right]^{-1} \\
& P_{k}=P_{k}^{-}-K_{k} C_{k} P_{k}^{-}
\end{aligned}
$$

where $\underline{\hat{x}}_{k}^{-}=\hat{E}\left\{\underline{x}_{k} \mid \underline{y}_{k-1}, \underline{y}_{k-2}, \ldots, \underline{y}_{0}\right\}$ is an estimate of the state vector, in the $k^{t h}$ stage, using the observations $y_{0}$ to $\underline{y}_{k-1}$, and $\underline{\hat{x}}_{k}=\hat{E}\left\{\underline{x}_{k} \mid \underline{y}_{k}, \underline{y}_{k-1}, \ldots, \underline{y}_{0}\right\}$ is an estimate of this state vector after adding the $k^{\text {th }}$ observations $y_{k} . P_{k}^{-}$and $P_{k}$ are defined in the same manner to be the estimates of the covariance matrices, in the $k^{\text {th }}$ stage, before and after using the $k^{\text {th }}$ observation, respectively.

\section{MODIFICATION OF THE EKF STRUCTURE}

\section{A. EKF2 Algorithm}

McSharry et al. [3] have proposed a synthetic ECG generator, which is based on a nonlinear dynamic model. This model has several parameters, which makes it adaptable to many normal and abnormal ECG signals. Details of this model can be found in [3]. Sameni et al. [1] proposed a polar form of this dynamic model which has some benefits compared to the original equations. They also proposed an EKF algorithm (which we name "EKF2") which has two states (ECG signal and its phase) and two observations (noisy ECG signal and observed noisy phase). Discrete stateequations of this model is as follows:

$$
\left\{\begin{array}{l}
\varphi_{k}=\left(\varphi_{k-1}+\omega \delta\right) \bmod (2 \pi) \\
z_{k}=-\sum_{i} \delta \frac{\alpha_{i} \omega_{k}}{b_{i}^{2}} \Delta \theta_{i} \exp \left(-\frac{\Delta \theta_{i}^{2}}{2 b_{i}^{2}}\right)+z_{k-1}+\eta
\end{array}\right.
$$

where $\Delta \theta_{i}=\left(\varphi_{k}-\theta_{i}\right) \bmod (2 \pi), \eta$ is a random additive noise that models the inaccuracies of the dynamic model and the summation over $i$ is taken over the number of Gaussian functions used for modeling the shape of the desired ECG channel. Following the notation of (1), the system state and process noise vectors are defined as follows:

$$
\begin{aligned}
& \underline{x}_{k}=\left[\varphi_{k}, z_{k}\right]^{T} \\
& \underline{w}_{k}=\left[\alpha_{P}, \ldots, \alpha_{T}, b_{P}, \ldots, b_{T}, \theta_{P}, \ldots, \theta_{T}, \omega, \eta\right]^{T}
\end{aligned}
$$

and the process noise covariance matrix is given by $Q_{k}=$ $E\left\{\left(\underline{w}_{k}-\underline{\bar{w}}_{k}\right)\left(\underline{w}_{k}-\underline{\bar{w}}_{k}\right)^{T}\right\}$. In this model, the noisy ECG recordings are assumed to be the observations for the KF. In addition to it, the phase may also be added as a second observation. In this definition R-peak is always assumed to be located at $\theta=0$ and the ECG contents lying between two consecutive R-peaks are assumed to have a linear phase between 0 and $2 \pi$ (or $-\pi$ and $\pi$ ). So by simply detecting the R-peaks, phase observation is achieved [1]. Observations may be related to the state vector as follows:

$$
\begin{aligned}
& \Phi_{k}=\varphi_{k}+v_{1 k} \\
& s_{k}=z_{k}+v_{2 k}
\end{aligned}
$$

where $v_{k}=\left[v_{1 k}, v_{2 k}\right]^{T}$ is observation noise and $R_{k}=E\left\{\left(\underline{v}_{k}-\right.\right.$ $\left.\left.\underline{v}_{k}\right)\left(\underline{v}_{k}-\underline{v}_{k}\right)^{T}\right\}$ is its covariance matrix. Details of "EKF2" algorithm can be found in [1].

\section{B. EKF3 Algorithm}

In order to use the angular velocity information of ECG beats, following the proposal [7] to use the angular velocity as a state, we use angular velocity of ECG as a third state in our system and and then we introduce a third observation corresponding to this new state. So we propose "EKF3" algorithm which has three states and three observations. Regarding the small changes of the PQRST morphology during several cycles, we use a simple autoregressive (AR) model for angular velocity. State-equations of this model are as follows:

$$
\left\{\begin{array}{l}
\varphi_{k}=\left(\varphi_{k-1}+\omega_{k-1} \delta\right) \bmod (2 \pi) \\
z_{k}=-\sum_{i} \delta \frac{\alpha_{i} \omega_{k-1}}{b_{i}^{2}} \Delta \theta_{i} \exp \left(-\frac{\Delta \theta_{i}^{2}}{2 b_{i}^{2}}\right)+z_{k-1}+\eta \\
\omega_{k}=\beta \omega_{k-1}+u_{k}
\end{array}\right.
$$

where $u_{k}$ denotes noise of angular velocity. In this work, for simplicity we consider $\beta=1$ but in general other values which are very close to one can be examined. Following the notation of (1), the system state and process noise vectors are defined as follows:

$$
\begin{aligned}
& \underline{x}_{k}=\left[\varphi_{k}, z_{k}, \omega_{k}\right]^{T} \\
& \underline{w}_{k}=\left[\alpha_{P}, \ldots, \alpha_{T}, b_{P}, \ldots, b_{T}, \theta_{P}, \ldots, \theta_{T}, \eta, u\right]^{T}
\end{aligned}
$$

In order to set up an EKF model based on the nonlinear synthetic model of (9), it is necessary to have a linearized version of the model. Consequently, the state-equation of (9) requires linearization using (2) and (3). By defining

$$
\left\{\begin{array}{l}
\varphi_{k}=F_{1}\left(\varphi_{k-1}, \omega_{k-1}, k\right) \\
z_{k}=F_{2}\left(\varphi_{k-1}, z_{k-1}, \omega_{k-1}, \alpha_{i}, b_{i}, \theta_{i}, \eta, k\right) \\
\omega_{k}=F_{3}\left(\omega_{k-1}, u, k\right)
\end{array}\right.
$$

The following equations represent the linearized model with respect to the state variables $\varphi_{k}, z_{k}$ and $\omega_{k}$ :

$$
\begin{aligned}
& \frac{\partial F_{1}}{\varphi_{k-1}}=1, \frac{\partial F_{1}}{\partial z_{k-1}}=0, \frac{\partial F_{1}}{\partial \omega_{k-1}}=\delta \\
& \frac{\partial F_{2}}{\partial \varphi_{k-1}}=-\sum_{i \in\{P, Q, R, S, T\}} \delta \frac{\alpha_{i} \omega_{k-1}}{b_{i}^{2}}\left[1-\frac{\Delta \theta_{i}^{2}}{b_{i}^{2}}\right] \exp \left(-\frac{\Delta \theta_{i}^{2}}{2 b_{i}^{2}}\right) \\
& \frac{\partial F_{2}}{\partial z_{k-1}}=1, \frac{\partial F_{2}}{\partial \omega_{k-1}}=-\sum_{i \in\{P, Q, R, S, T\}} \delta \frac{\alpha_{i} \Delta \theta_{i}}{b_{i}^{2}} \exp \left(-\frac{\Delta \theta_{i}^{2}}{2 b_{i}^{2}}\right) \\
& \frac{\partial F_{3}}{\partial \varphi_{k-1}}=0, \frac{\partial F_{3}}{\partial z_{k-1}}=0, \frac{\partial F_{3}}{\partial \omega_{k-1}}=1
\end{aligned}
$$


Similarly, the linearization of (11) with respect to the process noise components yields:

$$
\begin{aligned}
& \frac{\partial F_{1}}{\partial \alpha_{i}}=\frac{\partial F_{1}}{\partial b_{i}}=\frac{\partial F_{1}}{\partial \theta_{i}}=\frac{\partial F_{1}}{\partial \eta}=\frac{\partial F_{1}}{\partial u}=0 \\
& \frac{\partial F_{2}}{\partial \alpha_{i}}=-\delta \frac{\omega_{k-1} \Delta \theta_{i}}{b_{i}^{2}} \exp \left(\frac{-\Delta \theta_{i}^{2}}{2 b_{i}^{2}}\right) \\
& \frac{\partial F_{2}}{\partial b_{i}}=2 \delta \frac{\alpha_{i} \omega_{k-1} \Delta \theta_{i}}{b_{i}^{3}}\left(1-\frac{\Delta \theta_{i}^{2}}{2 b_{i}^{2}}\right) \exp \left(\frac{-\Delta \theta_{i}^{2}}{2 b_{i}^{2}}\right) \\
& \frac{\partial F_{2}}{\partial \theta_{i}}=\delta \frac{\alpha_{i} \omega_{k-1}}{b_{i}^{2}}\left(1-\frac{\Delta \theta_{i}^{2}}{b_{i}^{2}}\right) \exp \left(\frac{-\Delta \theta_{i}^{2}}{2 b_{i}^{2}}\right), \quad \frac{\partial F_{2}}{\partial \eta}=1, \quad \frac{\partial F_{2}}{\partial u}=0 \\
& \frac{\partial F_{3}}{\partial \alpha_{i}}=\frac{\partial F_{3}}{\partial b_{i}}=\frac{\partial F_{3}}{\partial \theta_{i}}=\frac{\partial F_{3}}{\partial \eta}=0, \frac{\partial F_{3}}{\partial u}=1
\end{aligned}
$$

In this model, we define three observations corresponding to the states. In order to estimate the angular frequency $\omega(t)$, a simple estimate would be $\omega(t)=\frac{2 \pi}{T(t)}$; where $T(t)$ is the R-R peak period in each ECG cycle. We only consider $\omega$ is related to the Heart Rate Variability (HRV) of the ECG signal and is known to be influenced by other physiological systems of the body. Some authors have worked on the spectral specifications of the HRV [9]; this suggests that $\omega$ itself may be assigned a dynamic model. Angular velocity observation is achieved by differentiating phase in each R-R peak period. As phase varies linearly between $-\pi$ and $\pi$ in each R-R peak period, $\omega$ is almost constant in this period but it may be contaminated by noise. Hence observations may be related to the state vector as follows which $v_{k}=\left[v_{1 k}, v_{2 k}, v_{3 k}\right]^{T}$ is observation noise:

$$
\begin{aligned}
& y_{k}=\left[\Phi_{k}, s_{k}, \Omega_{k}\right]^{T} \\
& \Phi_{k}=\varphi_{k}+v_{1 k} \\
& s_{k}=z_{k}+v_{2 k} \\
& \Omega_{k}=\omega_{k}+v_{3 k}
\end{aligned}
$$

\section{EKF3-2 Algorithm}

In subsection III-B, we used $\omega_{k}$ as a third state following the proposed idea in [7] but we defined three observations corresponding to three states. In this section, we propose "EKF3-2" algorithm which have three states same as "EKF3" algorithm but here we consider only two noisy observations, corresponding to the state variables $\varphi_{k}$ and $z_{k}$. In fact in this model, we did not have any corresponding observation for $\omega_{k}$ and we consider it as a hidden state and want to evaluate the results and compare them with results of "EKF3" algorithm. Here, state vector $\underline{x}_{k}$ and observation $\underline{y}_{k}$ are as follows:

$$
\underline{x}_{k}=\left[\varphi_{k}, z_{k}, \omega_{k}\right]^{T}, \quad \underline{y}_{k}=\left[\Phi_{k}, s_{k}\right]^{T}
$$

\section{RESUlTS}

The MIT-BIH Normal Sinus Rhythm Database [10] was used to study the performance of the proposed method. From this database, 30 seconds ECG signals of 10 different subjects without considerable artifacts which recorded at a sampling rate of $128 \mathrm{~Hz}$ were used. "EKF2", "EKF3" and "EKF3-2" algorithms were implemented in MATLAB. The performance of these algorithms were influenced by the initial value of the state vectors, as well as the covariance matrices of the process and measurement noise. Hence, we employ the initialization procedure described in [1]. MATLAB codes used for implementing "EKF2" algorithm were part of OSET [11] (Open-Source Electrophysiological Toolbox) which was proposed by Sameni et. al [1]. For evaluating the performance of the proposed algorithms, we compared the improvement in SNR by the means of expression:

$$
i_{m p}[d B]=S N R_{\text {out }}-S N R_{\text {in }}=10 \log \left(\frac{\sum_{i}\left|x_{n}(i)-x(i)\right|^{2}}{\sum_{i}\left|x_{d}(i)-x(i)\right|^{2}}\right)
$$

where $x$ denotes the clean ECG, $x_{d}$ is the denoised signal and $x_{n}$ represents the noisy ECG.

In order to investigate the performance of our algorithm and to compare it with different methods, we have implemented the previously introduced EKF model with 2 state variables (EKF2).

To ensure the consistency of the results, the whole procedure was repeated over the 30 seconds of the 10 ECG signals (records No. 16265, 16272, 16273, 16420, 16483, 16539, 16773, 16789, 17052 and 17453), each time using a different set of random white additive noise at the input. The filter output SNR calculation was averaged over the whole results for each input SNR. So results are independent of ECG record.

For a quantitative comparison, the mean of the SNR improvements versus different input SNRs are plotted in figure 1 and also presented in table I. In this figure, we can see that in all algorithms, SNR improvement is higher for low input SNRs. These results are achieved after averaging the results of all records with different noises.

SNR improvements versus different input SNRs of 4 signals are presented in figures 2, 3, 4 and 5. Results obtained with EKF3 and EKF3-2 are better than EKF2 especially in lower input SNRs. It proves the interest of considering angular velocity in the model.

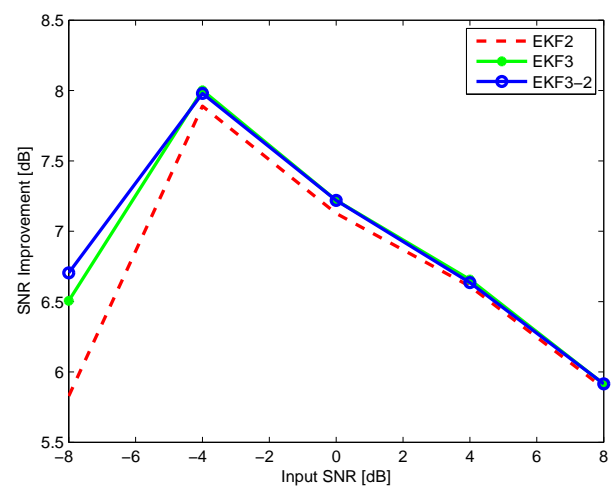

Fig. 1. Mean of SNR Improvement vs. Input SNR for all records.

TABLE I

MEAN OF SNR IMPROVEMENT IN DECIBELS FOR DIFFERENET ECG RECORDS OF MIT-BIH DATABASE

\begin{tabular}{|l|c|c|c|c|c|}
\hline Aalgorithm & SNRi=-8 & SNRi=-4 & SNRi=0 & SNRi=4 & SNRi=8 \\
\hline EKF2 & 5.829 & 7.89 & 7.127 & 6.635 & 5.915 \\
\hline EKF3 & 6.505 & 8 & 7.22 & 6.657 & 5.914 \\
\hline EKF3-2 & 6.704 & 7.98 & 7.22 & 6.61 & 5.886 \\
\hline
\end{tabular}




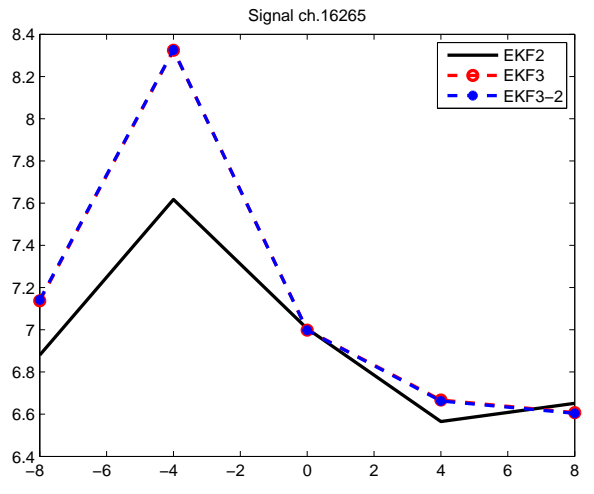

Fig. 2. SNR Improvement vs. Input SNR (Record No.16265).

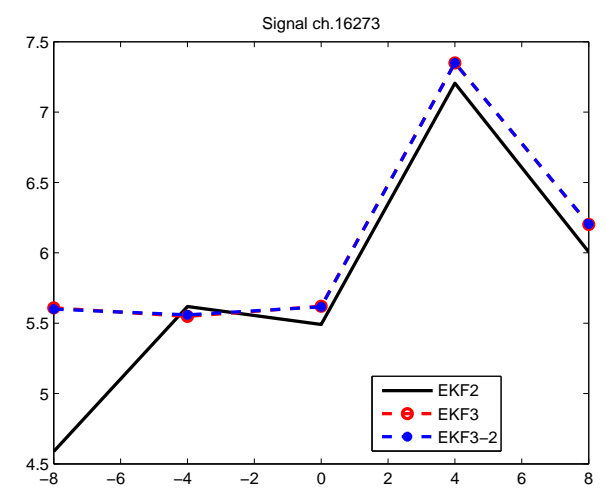

Fig. 3. SNR Improvement vs. Input SNR (Record No.16273).

\section{Discussion AND CONClusions}

Following the proposal [7] to use the angular velocity as a state, we have used angular velocity of ECG as a third state in our system. New proposed model is constructed based on having or not having the " $\omega$ " observation. We have introduced the latent (hidden) structure in EKF3-2 and the observable construction of this model as EKF3. Analysis of computational complexity of EKF3 and EKF3-2, which are extentions to the previous EKF2 shows that these algorithms are at the same level of cost. Quantitative evaluation of the algorithm shows the effect of new modifications on the estimate of the clean ECG.

Moreover, comparing to benchmark denoising schemes such as EKF2 algorithm, EKF3 and EKF3-2 bring more SNR improvement, especially in lower input SNRs. In "EKF3-2", although we did not have any corresponding observation for $\omega_{k}$, one achieved the same results as with the "EKF3" which is a nice property of the simplified model "EKF3-2".

Further work can include the use of this proposed method for more real ECG datasets especially abnormal signals. It is also of interest to find other applications except denoising which needs angular velocity of ECG.

\section{ACKNOWLEDGMENT}

The authors would like to thank Dr Reza Sameni and Dr Omid Sayadi, for their helpful comments on the paper.

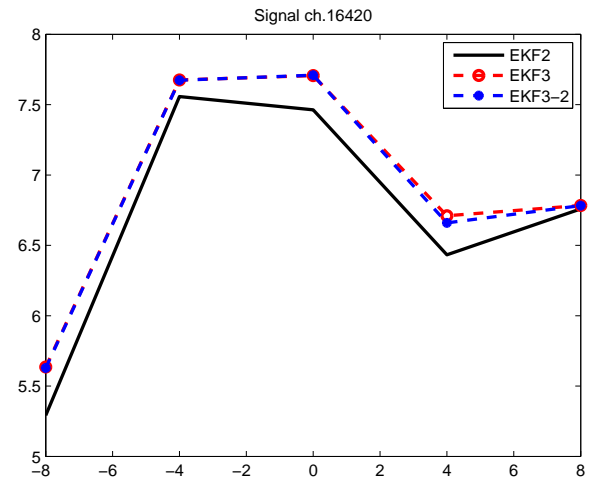

Fig. 4. SNR Improvement vs. Input SNR (Record No.16420).

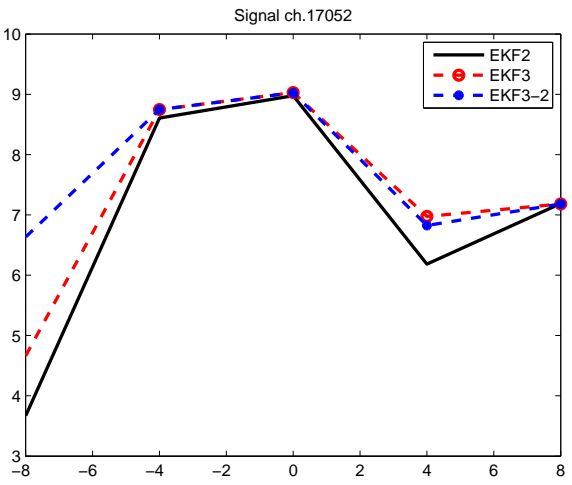

Fig. 5. SNR Improvement vs. Input SNR (Record No.17052).

\section{REFERENCES}

[1] R. Sameni, M. B. Shamsollahi, C. Jutten, and G. D. Clifford, "nonlinear bayesian filtering framework for ECG denoising," IEEE Trans. Biomed. Eng., vol. 54, no. 12, pp. 2172-2185, Dec. 2007.

[2] R. Sameni, M. B. Shamsollahi, and C. Jutten, "Model-based bayesian filtering of cardiac contaminants from biomedical recordings," Physiol. Meas., vol. 29, pp. 595-613, 2008.

[3] P. E. McSharry, G. D. Clifford, L. Tarassenko, and L. A. Smith, "A dynamicmodel for generating synthetic electrocardiogram signals," IEEE Trans. Biomed. Eng., vol. 50, no. 3, pp. 289-294, Mar. 2003.

[4] G. D. Clifford, A. Shoeb, P. E. McSharry, and B. A. Janz, "Modelbased filtering, compression and classification of the ECG," Int. J. Bioelectromagn., vol. 7, no. 1, pp. 158-161, 2005.

[5] O. Sayadi and M. B. Shamsollahi, "ECG denoising and compression using a modified extended kalman filter structure," IEEE Trans. Biomed. Eng., vol. 55, no. 9, pp. 2240-2248, Sep. 2008.

[6] O. Sayadi and M. B. Shamsollahi, "Synthetic ECG generation and bayesian filtering using a gaussian wave-based dynamical model," Physiol. Meas., vol. 31, pp. 1309-1329, 2010.

[7] C. Lin, M. Bugallo, C. Mailhes, and J. Y. Tourneret, "ECG denoising using a dynamical model and a marginalized particle filter," in IEEE Asilomar Conf. on Signals, Systems and Computers, Pacific Grove, CA, USA, Nov.2011.

[8] S. M. Kay, Fundamentals of statistical Signal Processing:Estimation Theory, Prentice Hall PTR, 2003.

[9] Task Force of the European Society of Cardiology, the North American Society of Pacing, and Electrophysiology, "Heart rate vriability : Standards of measurement, physiological interpretation, and clinical use," European Heart Journal., pp. 354-381, 1996.

[10] MIT-BIH Normal Sinus Rhythm Database [online], Available:http://www.physionet.org/physiobank/database/nsrdb/.

[11] Open-Source Electrophysiological Toolbox [online], Available: www.oset.ir. 